\title{
High Resolution Visualization of the Articular Cartilage Collagen Network by Helium Ion Microscopy
}

\author{
W.S. Vanden Berg-Foels, ${ }^{*}$ L. Scipioni, ${ }^{* *}$ C. Huynh, ${ }^{* *}$ and X. Wen* \\ * Clemson University \& Medical University of South Carolina, Charleston, SC 29425 \\ ** Carl Zeiss NTS, Peabody, MA 01960
}

INTRODUCTION: Articular cartilage lines the ends of long bones in the joints of the body, providing shock absorption and a smooth, lubricated surface for low friction movement. Cartilage degeneration (arthritis) is the leading cause of adult disability in the United States [1]. Cartilage is primarily composed of an extracellular matrix, the composition of which determines the tissue mechanical properties [2]. Two key matrix components are 1) polysaccharide macromolecule complexes (proteoglycans) that attract water and cause swelling and 2) a 3D collagen fibril network that resists the proteoglycan swelling pressure. One of the early signs of degeneration is cartilage swelling due to disruption of the collagen network.

The ability to characterize the spatial organization of the collagen network is necessary for the study of degeneration mechanisms, the assessment the efficacy of drug treatment to modify degeneration progression, and the evaluation of tissue engineering efforts to regenerate durable articular cartilage. Available imaging modalities have enabled either macroscopic tissue visualization or a high resolution evaluation of a very small region. To our knowledge, there is no previous research that provides a cohesive characterization of the organization of the $3 \mathrm{D}$ collagen network from the macroscopic tissue level down to the nanoscopic level.

Helium ion microscopy (HIM) was selected to investigate the articular cartilage collagen network. HIM has several advantages over other modalities, including 1) a resolution range that enables evaluation of the network from the macroscopic ( $1 \mathrm{~mm}$ field of view) down to the sub-nanometer scales, 2) an extended depth of field that provides a focused image of 3D features, 3) enhanced contrast for better visualization of surface features, 4) minimal sample damage, and 5) charge control so that gold film coating of insulating samples is unnecessary [3, 4]. The results presented here are a first step towards a more complete understanding of the articular cartilage collagen network.

METHODS: Osteochondral strips were harvested from the knee patellar groove of rabbits that were humanely euthanized for other research. Proteoglycans were enzymatically digested from the matrix without disrupting the collagen network. The samples were dehydrated in a graded alcohol series and dried. Images were acquired with an Orion Plus ${ }^{\circledR}$ HIM (Carl Zeiss NTS, LLC, Peabody, MA, USA) operated in secondary electron mode with an acceleration voltage of $31.4 \mathrm{kV}$ and a beam current of 0.4-0.6 pA. Charge buildup was neutralized with an electron flood gun.

RESULTS AND CONCLUSIONS: The resolution range of the Orion HIM enabled visualization from the full thickness of the cartilage layer down to the banding of the type 2 collagen (FIG. 1). Variation in matrix orientation could be clearly distinguished, with fibrils oriented parallel to the articular surface in the subsurface layer and perpendicular to the surface in the deeper inter-cellular matrix. The difference in collagen fibril organization between the peri-cellular and inter-cellular matrices was also apparent. The many small fibrils visible are likely collagens known to link the 
larger type 2 fibrils. Our results demonstrate the potential of HIM to advance our understanding of the articular cartilage collagen network in health and disease.

References

[1] United States Bone and Joint Decade: The Burden of Musculoskeletal Diseases in the United States, American Academy of Orthopaedic Surgeons, Rosemont, IL, 2008.

[2] A.J. Grodzinsky, CRC Crit Rev Bioeng. 9 (1983) 133.

[3] B.W. Ward et al., J. Vac. Sci. Technol. B. 24 (2006) 2871.

[4] D.C. Bell, Microsc. Microanal. 15 (2009) 147.

[5] This research was supported by NIDCR T32 DE017551 (WSVBF).

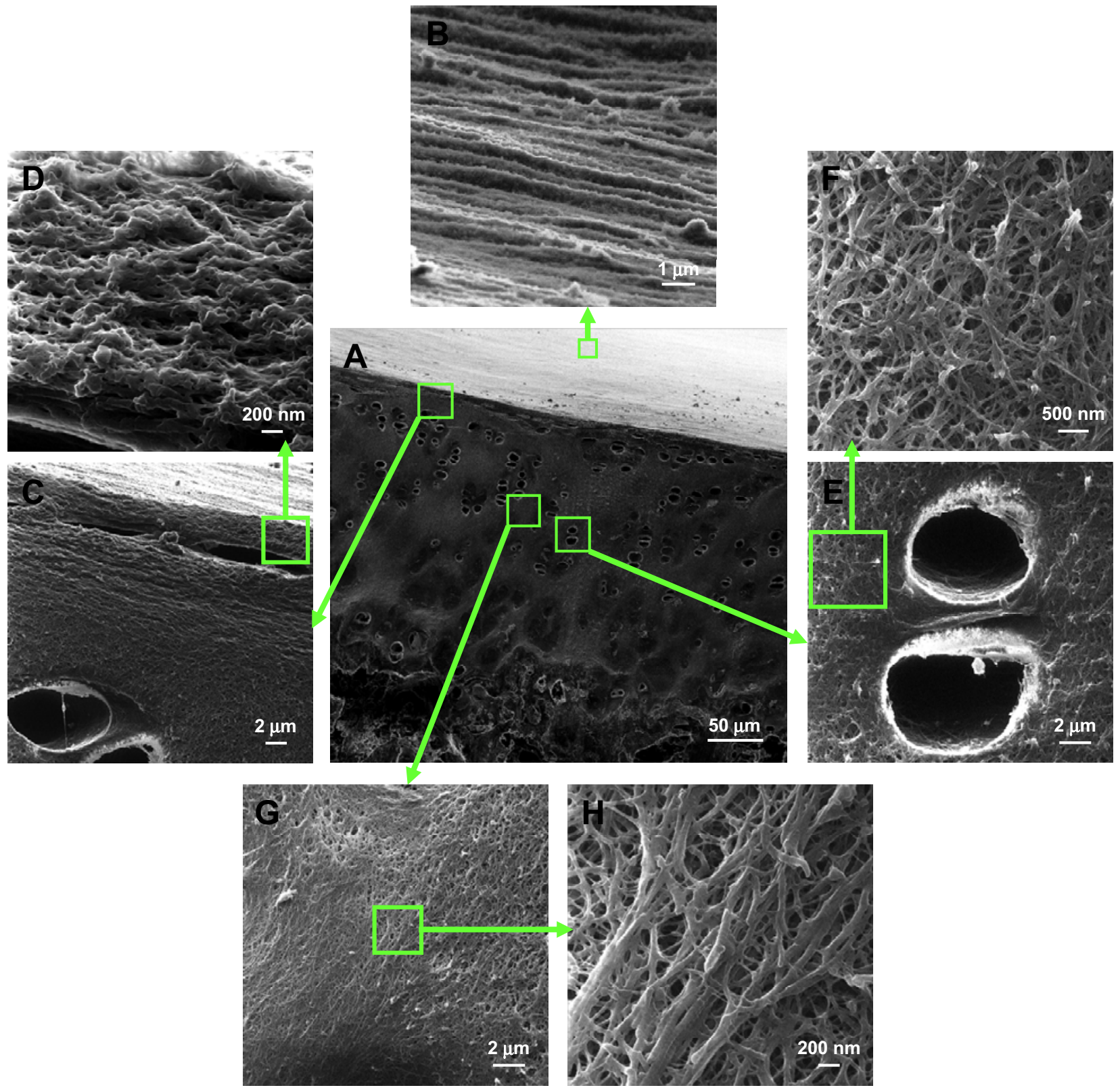

FIG 1. Survey of spatial variation in collagen network organization: A) cartilage cross-section; B) articular surface; C, D) subsurface; E, F) peri-cellular matrix; G, H) inter-cellular matrix. 